\title{
LA ENSEÑANZA DEL DERECHO AGRARIO EN CUBA: NUEVAS REFLEXIONES Y PROPUESTAS DE CAMBIO
}

\author{
THE TEACHING OF AGRARIAN LAW IN CUBA: NEW REFLECTIONS AND PROPOSALS FOR \\ CHANGE
}

\author{
Dr. Rolando Pavó Acosta** \\ Msc. Daniel Bello Vázquez \\ Msc. Juan Ramón Pérez Carrillo
}

\begin{abstract}
Resumen
Resulta apreciable la pertinacia de los criterios negativos en torno a la autonomía didáctica del Derecho Agrario, lo que de manifiesto la sobrevivencia aún de ciertos niveles de incomprensión, incluso de desconocimiento con respecto a las esenciales razones económicas, políticas, sociales y jurídicas que han argumentado a través de casi un siglo, sólidamente, la existencia del Derecho Agrario como ciencia y como rama jurídica, lo que motiva la necesidad de continuar investigando sobre la enseñanza del Derecho Agrario, evaluando los aciertos y carencias en el desarrollo de la impartición de esta materia dentro de los estudios jurídicos. El presente trabajo, ofrece una semblanza de los principales programas analíticos de la asignatura de Derecho Agrario para los diferentes modelos pedagógicos (presencial, semipresencial y a distancia) presentando un diagnóstico acerca de cuáles han sido las regularidades, aciertos y las carencias sobre las cuales es preciso continuar trabajando. Los principales resultados que aquí se presentan, además del aludido diagnóstico, consisten en la propuesta fundamentada de continuar defendiendo la impartición del Derecho Agrario como asignatura autónoma y en un conjunto de recomendaciones encaminadas al perfeccionamiento de dichos programas.
\end{abstract}

Palabras clave: Derecho Agrario; Enseñanza; Autonomía didáctica.

\begin{abstract}
The pertinacity of the negative criteria regarding the teaching autonomy of Agrarian Law is appreciable, which further demonstrates the survival of certain levels of misunderstanding, even ignorance, regarding the essential economic, political, social and legal reasons that have argued through nearly a century, solidly, the existence of Agrarian Law as a science and legal branch, which motivates the need for further research on the teaching of Agrarian Law, assessing the strengths and the weaknesses in the development of teaching this subject within legal studies. This paper provides a sketch of the main analytical programs the subject of Agrarian Law for different pedagogical models (classroom, blended learning and distance learning) presenting a diagnosis about what were the regularities, the successes and the shortcomings, that it is necessary continue working about. The main results here, besides the aforementioned diagnosis, present consist grounded proposal to continue defending the teaching of Agrarian Law as an autonomous subject and a set of recommendations aimed at improving these programs.

Keywords: Agrarian Law; Education; Teaching autonomy.

\footnotetext{
* Título en inglés y abstract por Wendel Rosa Borges.

** Doctor en Ciencias Jurídicas, Profesor de la Facultad de Derecho, Universidad de Oriente, Santiago de Cuba.

*** Licenciado en Derecho por la Facultad de Derecho de la Universidad de Oriente, Santiago de Cuba. Profesor del Departamento de Derecho, Facultad de Ciencias Sociales y Humanísticas, Universidad de Granma, Cuba.
} 


\section{Introducción}

La persistencia de las polémicas en el ámbito nacional acerca de la autonomía didáctica del Derecho Agrario, la intermitencia de los debates entre partidarios y detractores, y especialmente la sugerencia vertida en el contexto de una de las reuniones recientes de la Comisión Nacional de la Carrera de Derecho, de considerar al Derecho Agrario como una asignatura optativa en el nuevo Plan de Estudios D - en proceso de implantación en estos momentos-, constituyen buenas razones para el examen acerca de los logros y desaciertos experimentados en cuanto al desarrollo de la Didáctica Especial de esta asignatura y de su Programa Analítico, luego de 25 años en que fue consagrada su autonomía didáctica al implantarse el denominado Plan de Estudios B, a inicios de la década de 1980.

Resulta apreciable la pertinacia de los criterios negativos en torno a la autonomía didáctica del Derecho Agrario, lo que de manifiesto la sobrevivencia aún de ciertos niveles de incomprensión, incluso de desconocimiento con respecto a las esenciales razones económicas, políticas, sociales y jurídicas que han argumentado a través de casi un siglo, sólidamente, la existencia del Derecho Agrario como ciencia y como rama jurídica. Ello refleja además un limitado conocimiento acerca del desarrollo doctrinal, normativo y didáctico de esta materia a escala internacional y muy especialmente en el ámbito continental, y también pudiera estar presente la recepción de esquemas y concepciones procedentes de Europa con respecto al diseño curricular de la carrera de Derecho, donde a pesar de ser en ese continente donde se comienza a forjar la autonomía científica del Derecho Agrario alrededor de 1920-30, ciertamente en muchas universidades ésta no se ha impartido como disciplina autónoma. ${ }^{1}$

Es que a pesar del peso incuestionablemente importante de la actividad agropecuaria para el desarrollo económico y social de muchos países, persisten criterios que ven en el Derecho Agrario sólo a una rama legislativa, a un conjunto de normas jurídicas. Ello explicaría en el caso de Cuba, la circunstancia de cierto atraso en el desarrollo científico de esta disciplina, hecho que deviene causa y efecto de toda otra serie de fenómenos: en las publicaciones jurídicas nacionales se le dedica mucho menos espacios a los temas agrarios que a otras materias, la Sociedad Científica de Derecho Agrario, ha resultado de creación reciente, hasta el presente se han elaborado solamente dos tesis de doctorado sobre Derecho Agrario y la cifra de tesis de maestría resulta relativamente baja. ${ }^{2}$

R. Fac. Dir. UFG, V. 37, n. 1, p. 117 - 142, jan. / jun. 2013

ISSN 0101-7187 
Lo curioso del asunto es que existen en el país miles de juristas, que como asesores, abogados o consultores en materia agraria prestan sus servicios jurídicos a las dependencias nacionales y territoriales del Ministerio de la Agricultura, Ministerio del Azúcar y de la Asociación Nacional de Agricultores Pequeños, a las empresas y uniones empresariales pertenecientes a estos organismos y a las Cooperativas Agropecuarias. Hay provincias del país en las que por su composición sociodemográfica y la estructura de su economía, la conflictividad y la litigiosidad más esencial, que se manifiesta ante la jurisdicción de las Salas de lo Económico de los Tribunales y las de las Salas y Secciones de lo Civil y las reclamaciones y quejas ante el Gobierno y las dependencias administrativas, tiene que ver con la propiedad agraria y con actividad agropecuaria. ${ }^{3}$

Es preciso apuntar en tal sentido que a los estudios de la carrera de Derecho, que se venían desarrollando mediante el modelo de enseñanza de los cursos regulares diurnos, en las cuatro universidades principales que había en el país hasta los años 90, se vino a sumar la implantación gradual, a partir del año 2000, de los estudios por esta misma modalidad, en todas las capitales de provincia del país, a lo que agrega la implementación de los estudios universitarios de Derecho a través del denominado Modelo de la Universalización, mediante la modalidad semipresencial en cada uno de los municipios del país.

Ya ubicados en este contexto, la presente exposición ha tenido como objetivos:

1) Partiendo de los fundamentos que argumentan la autonomía didáctica del Derecho Agrario, identificar los avances experimentados en el desarrollo de la enseñanza del Derecho Agrario en Cuba.

2) Identificar los logros y las deficiencias apreciables en lo relativo al desarrollo de una Didáctica Especial del Derecho Agrario y de la concepción del Programa Analítico de la Asignatura en sus diferentes modelos pedagógicos.

3) Proponer un conjunto de recomendaciones encaminadas al perfeccionamiento del Programa de la Asignatura y de su Didáctica Especial en los diferentes modelos pedagógicos.

\section{Los Fundamentos de la Autonomía Didáctica del Derecho Agrario. Aciertos y Carencias en el Desarrollo de los Programas Analíticos para la Enseñanza Presencial en Cuba}

R. Fac. Dir. UFG, V. 37, n. 1, p. 117 - 142, jan. / jun. 2013

ISSN 0101-7187 


\subsection{El Derecho Agrario: Los argumentos a favor de su autonomía didáctica ${ }^{4}$}

En Italia existen desde hace varias décadas, experiencias en la concepción del Derecho Agrario como disciplina autónoma impartiéndose así como parte de los estudios en las Facultades de Jurisprudencia, en la Facultades de Agrícolas y en las de Ciencias Económicas y Comerciales. En España se ha venido impartiendo como materia autónoma en varios centros de educación superior, especialmente en las Escuelas de Ingenieros Agrónomos, un gran propulsor de esos avances ha sido el profesor Alberto Ballarín y Marcial, pero también en algunas facultades de Derecho. ${ }^{5}$

Se observan indiscutiblemente los mayores avances en la independización didáctica del Derecho Agrario, en América Latina, apreciándose en ello un superior nivel en ese logro en el caso de México, donde ello se reconoció oficialmente desde 1939. Resulta notable también el desarrollo que ha alcanzado su didáctica como materia independiente tanto en las Facultades de Derecho como de Ciencias Agrícolas de Perú, Colombia, Uruguay, Paraguay, Costa Rica, Venezuela, en este último país además de impartirse como asignatura en pregrado, desde hace ya una década existe el Instituto Iberoamericano de Derecho Agrario y Reforma Agraria (IIDARA) con sede en la Universidad de Los Andes, Mérida, que ha realizado una labor trascendente en la organización de eventos internacionales, cursos de postgrado, el desarrollo de estudios legislativos y otras labores de relevancia en este campo. En Uruguay existe una extensa tradición en la impartición universitaria del Derecho Agrario, en la década del 50 en la Facultad de Derecho y Ciencias Sociales de Montevideo el Centro de Estudios de Derecho Rural y la Cátedra de Derecho Rural, dedicada inicialmente a los cursos de postgrado, luego en 1982 se constituye el Instituto Uruguayo de Derecho Agrario, adscrito a la referida Facultad. ${ }^{6}$

Aunque no se puede negar los logros alcanzados en este sentido, se advierte que los avances no han sido uniformes, como es el caso de Argentina donde por una parte, se imparte el Derecho Agrario en las Universidades de La Plata, El Litoral y el Rosario, si bien en algún momento se impartió también en las de Córdoba y Santa Fe, y por otro lado en el resto del país el contenido de la materia agraria se diluye en una disciplina denominada, Derecho de los recursos naturales, Régimen Jurídico de los Recursos Naturales o en el Derecho Ambiental. ${ }^{7}$ 
Tal y como aparecen resumidos en la obra de M. Ruiz Massieu, los argumentos positivos más comunes, a favor de la independencia didáctica del Derecho Agrario son los siguientes: ${ }^{8}$

1.- La necesidad de dotar a los egresados de un conocimiento técnico jurídico especializado en materia agraria, dada la trascendencia del sector agrario.

2.- La imposibilidad de que su estudio fragmentado pueda conducir al conocimiento cabal de los principios ejes de la materia.

3.- La especial consideración y rigor que se ha logrado al impartirse la materia en los centros universitarios.

Además de ello, las experiencias acumuladas permiten advertir que allí donde el contenido de la materia agraria se diluye entre otras instituciones del Derecho Civil, enseñándose la materia agraria de modo fragmentado y carente de unidad orgánica y sistemática, las consecuencias nocivas más apreciables residirían en que:

a) No se desarrollaría consecuentemente la docencia en su interrelación con el desarrollo de investigaciones científicas en la materia y al no haber un medio científico propicio para el desarrollo doctrinal y de la didáctica especial de esta materia, descendería el rigor técnico en la impartión de esta disciplina.

b) Se corre el riesgo de interferencias nocivas al extrapolarse instituciones y principios del Derecho Civil al Derecho Agrario y de desnaturalizarse en su interpretación las normas de Derecho Agrario, y producto de ello las Universidades no podrían cumplir su cometido de formar intérpretes de la legislación agraria.

c) Se comprometería y afectaría, el proceso de formación de Profesores de la materia y finalmente, difícilmente se lograrían formar los especialistas en la materia, con la calificación que demanda la sociedad.

Las Universidades históricamente han tenido el cometido de dar respuesta a las diferentes demandas sociales: formar a los especialistas con el nivel que se requiere, ahondar científicamente en los problemas que interesan a la sociedad, y a partir de sus resultados ayudar al perfeccionamiento institucional y legislativo. Esa tarea sólo la pueden cumplimentar los claustros universitarios y ninguna otra institución. Pero las Universidades no pueden cumplir ese cometido tan importante si se estancan científicamente, si se quedan por debajo de su tiempo, y esto en nuestra opinión es el riesgo que se R. Fac. Dir. UFG, V. 37, n. 1, p. 117 - 142, jan. / jun. 2013 
asume allí donde el estudio del régimen jurídico de la materia agraria se aborda en su mayor parte integrado al Derecho Civil.

Diferentes Organizaciones y organismos de las Naciones Unidas y organizaciones regionales se han manifestado reiteradamente a favor de la autonomía didáctica de la materia agraria. Ya en la XII Conferencia Mundial de la FAO, efectuada en Roma, en su Resolución No.17/63, se emitió una recomendación general a ese respecto y en otras reuniones, tales como la $\mathrm{V}$ Conferencia Interamericana de Agricultura y la VI Conferencia Regional de la FAO para América Latina, se recomendó a los gobiernos prestar la máxima atención a la formación de especialistas en legislación agraria y a las Universidades les señalaba la necesidad de dar impulso a los estudios de Derecho Agrario como disciplina especial y autónoma. ${ }^{9}$

Coincidiendo con esa propuesta en la VI Conferencia de la UDUAL, efectuada en Bogotá Colombia, efectuada del 26 de septiembre al 1ro de octubre de 1976, se adoptó como acuerdo que recomienda a todas las Facultades de Derecho de América Latina:

Que dispongan la creación de cátedras obligatorias de Derecho Agrario, donde no las hubiera, cuyo ámbito abarcará especialmente todas las situaciones que se derivan de la relación hombre-tierra, la conservación de recursos naturales renovables y del medio ambiente. ${ }^{10}$

\subsection{La Introducción de la asignatura de Derecho Agrario en el Plan de Estudios de la carrera de Derecho}

Resulta necesario precisar que en el año 1982, comienza la implantación del Plan de Estudio B y que como una de sus consecuencias, se introduce la implantación del Derecho Agrario como una nueva asignatura. ${ }^{11}$

Los antecedentes de la autonomía didáctica del Derecho Agrario en Cuba, deben ubicarse, primeramente, en la labor del Dr. Manuel Dorta Duque, profesor de Derecho Hipotecario en la Universidad de La Habana. Es así que el 9 de julio de 1943, la Facultad de Derecho de esta Universidad, a iniciativa de la referida Cátedra de Derecho Hipotecario adoptó un acuerdo, que luego fuera ratificado por el Consejo Universitario, relativo a la Introducción de un curso dedicado al estudio 
de la legislación agraria, lo que deviniera luego en la fundación de la primera Cátedra de Derecho Agrario en Cuba. ${ }^{12}$

En la década del 50, se impartió el Derecho Agrario en los estudios de la carrera de Derecho desarrollados en las Universidades de Villanueva, la José Martí y la Masónica. ${ }^{13}$

Luego en 1960, el profesor Dr. Orestes Hernández Más, también en la Universidad de la Habana, condujo la impartición de la asignatura de Derecho Agrario, al reabrirse la carrera de Derecho, el cual también produjo unos folletos mimeografiados.

Un poco tardíamente, es decir, en los primeros años de la década de los 80 al ponerse en vigor los llamados Planes de Estudio "B" por el Ministro de la Educación Superior, es que se introduce en pregrado, la asignatura de Derecho Agrario en la carrera de Derecho. ${ }^{14}$

El calificativo de tardío deriva de que hacía ya 20 años que se había dictado la Reforma Agraria, habiéndose acumulado un material normativo extenso sobre la materia agraria que independizaba legislativamente al Derecho Agrario del Derecho Civil y por otra parte desde el propio año 1959, se había instituido un mecanismo administrativo para el conocimiento de buena parte de los conflictos en materia.

\subsection{La ubicación de la asignatura en el Plan de Estudios}

La ubicación de la asignatura dentro del currículo de la carrera de Derecho a través de los diferentes Planes de Estudio, y la concepción de la Universidad de Oriente y la de La Habana, ha sufrido variaciones, como podrá verse en la Tabla 1.

Tabla 1. Ubicación del Derecho Agrario en relación con los Planes de Estudio

\begin{tabular}{|c|c|c|c|}
\hline $\begin{array}{c}\text { Plan de } \\
\text { Estudios }\end{array}$ & Universidad & Año Académico & Semestre \\
\hline B & UH & III & 5 \\
\hline
\end{tabular}

R. Fac. Dir. UFG, V. 37, n. 1, p. 117 - 142, jan. / jun. 2013

ISSN 0101-7187 


\begin{tabular}{|l|l|l|l|}
\hline B & UO & IV & 8 \\
\hline C & UH & V & 9 \\
\hline C & UO & III & 6 \\
\hline C Perfeccionado & UH & IV & 8 \\
\hline C Perfeccionado & UO & IV & 8 \\
\hline
\end{tabular}

Salta a la vista la itinerancia o movilidad de la asignatura dentro del currículo, lo que pudiera estar asociado a muy diversos factores.

- Insuficientes experiencias acumuladas en la impartición de la asignatura.

- Diversidad de criterios entre las diferentes universidades, acerca de los enfoques y contenidos de la enseñanza de Derecho.

La polémica a este respecto tuvo su clímax con motivo de la implantación del Plan de Estudios C, en 1991, pues la Resolución 288 de 1988, del Ministro de la Educación Superior, Reglamento Docente Metodológico, concedió facultades a los rectores de los Centros de Educación Superior, para establecer modificaciones a los Planes de Estudio de las carreras ya aprobados nacionalmente, a propuesta de las Comisiones de Carrera de las Facultades.

Resulta oportuno, hacer notar que desde finales de los 90 y en lo que va de la primera década del 2000, se fue gestando un nuevo contexto en el que ha existido mucho mayor nivel de búsqueda del consenso en lo que a la impartición del Derecho se refiere, concretándose en la realización de varias reuniones nacionales, de profesores que imparten las mismas asignaturas y una mayor sistematicidad en el funcionamiento de la Comisión Nacional de Carrera.

Bajo tales circunstancias se pudo arribar por consenso a la ubicación actual de la asignatura de Derecho Agrario, en el cuarto año de la carrera, lo que debe considerarse un acierto y con lo cual se resuelven en buena medida las opiniones divergentes de ambas universidades.

\subsection{EI Sistema de Habilidades}


El aspecto referido al sistema de habilidades, en el contexto del Programa Analítico de la asignatura, se encamina a anticipar cuáles son los cambios esperados en la formación de los estudiantes, que se habrían de producir con la impartición de la asignatura en cuestión, en lo que se refiere a capacidades, aptitudes profesionales, más allá de los alcances estrictamente gnoseológicos.

Precisamente, una de las innovaciones introducidas en la estructura del Plan C perfeccionado, fue la de formular el sistema de habilidades, por lo que puede considerarse este aspecto como de reciente aparición en los Programas de las asignaturas, a este respecto se estableció que el sistema de habilidades era uno de los aspectos de carácter preceptivo, indicativo, siendo acordados en la Comisión Nacional de Carrera, y que por tanto deben ser plasmados invariablemente, sin ningún cambio en todos los programas analíticos de todas las Universidades donde se imparta la asignatura.

Las habilidades son la expresión más concreta de lo que se quiere lograr con la impartición de la asignatura y por tanto se supone que en su formulación debe superarse el carácter abstracto y general de los objetivos. En tal virtud, es apreciable que no es del todo satisfactoria la redacción de algunas en el Programa, véase que por ejemplo que en el Sistema de Habilidades, la que se designa con el número 3, se enuncia, "Desarrollar habilidades y hábitos acordes con las concepciones teóricas y prácticas en que se fundamenta la legislación agraria", aquí resulta evidente la falta de un enunciado que exprese con claridad cuál es la habilidad concreta que se desea formar en el alumno que reciba la asignatura.

\subsection{El Sistema de Valores.}

Otra de las novedades introducidas en la concepción de los programas de las asignaturas con la implantación del Plan de Estudios C Perfeccionado, consiste en la exigencia de formular el sistema d valores que se espera formar o desarrollar en los alumnos, lo que constituye un discutible logro del Plan. En realidad la amplitud, importancia y diversidad del contenido del Derecho Agrario, ofrece envidiables posibilidades para la formación y desarrollo de valores en los alumnos a través de esta asignatura. 
Se ha insistido, con reiteración en la formulación del principio de que en el proceso docente educativo se debe educar a través de la instrucción y en muchos campos del saber el profesor encuentra mayores obstáculos para lograr la realización de ese principio y entonces el profesor tiene que forzar un poco el proceso de formación de determinados valores, sin embargo en el Derecho Agrario dados los contenidos, se facilitan el desarrollo de los procesos afectivos y orientativos de la conducta.

En la actual concepción del programa de la asignatura se ha arribado a una formulación bastante explícita sobre el sistema de valores y los espacios (por temas y actividades docentes concretas) en los cuales se trabajará en ellos.

\subsection{Indicaciones Metodológicas y Organizativas.}

De manera tradicional se ha incluido este aspecto en los Programas Analíticos de las asignaturas desde los Planes B, hasta los actuales. Es apreciable que por lo general, lo que se ha hecho es reiterar en esta parte de la redacción del Programa, aspectos ya tratados en otros epígrafes, como la ubicación de la asignatura, la cantidad de horas y su distribución por temas y actividades, las formas de docencia., el sistema de evaluación. De lo cual resulta que en este tópico, el Programa pudiera explicitar otras cuestiones, tales como:

Cuáles son los cambios o novedades que contiene el Programa, en relación con los anteriores y por qué se han introducido.

Cuáles son las peculiaridades didácticas de la asignatura que el alumno debe tomar en consideración para cumplir con los objetivos y lograr las habilidades previstas?

Cuáles orientaciones se considera pertinente impartir al alumno, sobre aspectos esenciales del contenido, en los cuáles debe prestar mayor atención para lograr el cumplimiento de los objetivos.

En cuáles temas y mediante cuáles actividades se aprovechará para trabajar en el cumplimiento de Estrategias Educativas específicas que se ejecutan en la carrera (antidrogas, de dominio de la Historia Nacional, de desarrollo de conocimientos y habilidades en el manejo de las Nuevas Tecnologías de la Informática y la Computación (NTIC), de dominio de idiomas extranjeros y otras) 


\subsection{Los Objetivos}

En el proceso de perfeccionamiento del Programa de la asignatura de Derecho Agrario en cuanto a este aspecto se aprecia como tendencias fundamental, que se han ido formulando Objetivos Generales de modo cada vez más abstractos y generales. Así que de 10 objetivos generales entre educativos e instructivos que se enunciaban en el Plan B, ya en el Plan C perfeccionado se plantean solo 3 en los cuales se integran propósitos instructivos y educativos, lo que nos parece muy positivo, pues revelan la intensión de lograr mayores niveles de materialización del principio didáctico de unidad de la instrucción y la educación, es decir que la actual formulación de los Objetivos Generales se proyecta hacia el propósito de educar a través de la instrucción, tal y como se ilustra en la Tabla 2.

Tabla 2. Número y Tipo de Objetivos

\begin{tabular}{|l|l|l|l|}
\hline \multicolumn{1}{|c|}{$\begin{array}{c}\text { Plan de } \\
\text { Estudios }\end{array}$} & \multicolumn{1}{c|}{$\begin{array}{c}\text { Objetivos } \\
\text { Educativos }\end{array}$} & $\begin{array}{c}\text { Objetivos } \\
\text { Instructivos }\end{array}$ & Total \\
\hline B UH & 4 & 6 & 10 \\
\hline B UO & 4 & 6 & 10 \\
\hline C UH & 4 & 3 & 7 \\
\hline C UO UH & - & 3 & 7 \\
\hline $\begin{array}{l}\text { C Uo } \\
\text { Perfeccionado }\end{array}$ & & - & 3 \\
\hline $\begin{array}{l}\text { C UO } \\
\text { Perfeccionado }\end{array}$ & & - & 3 \\
\hline
\end{tabular}

\subsection{Sistema de Conocimientos:}

No ha existido unanimidad en la concepción del sistema de conocimientos en los diferentes programas analíticos que ha tenido la asignatura en su impartición. A los efectos de lograr la mejor comprensión de estas variaciones sería necesario tomar en consideración algunos hechos: 
Entre el Plan de Estudio B y el actual Plan C perfeccionado, han mediado 25 años, durante los cuales se han experimentado avances notables en la doctrina del Derecho Agrario y se ha producido la recepción de conceptos y teorías procedentes de otros países. Cabe agregar que alrededor del objeto y contenido del Derecho Agrario, han tenido lugar interminables debates desde las primeras décadas del Siglo XX hasta nuestros días, no lográndose unanimidad de opiniones,

Conforme a lo acordado en las reuniones de la Comisión de la Carrera de Derecho, se llegó al consenso de que los Objetivos, Habilidades y Contenidos, tendrían un carácter directivo y por tanto serían invariables en los programas de la misma asignatura en las diferentes universidades.

Al iniciarse los estudios de Derecho Agrario, como asignatura autónoma, resulta evidente que apenas se contaba con un desarrollo doctrinal mínimo en torno a los temas que conforman la teoría general del Derecho Agrario (Objeto, contenido, métodos, fuentes, etc.), pues la literatura jurídica cubana en los 60 y hasta los 80, se concentraba en temas como la Reforma Agraria primeramente y luego, en las cooperativas agropecuarias. En ese momento fundacional del Derecho Agrario como asignatura, todavía muchos no lograban despojarse de la concepción del Derecho Agrario como subrama del Derecho Civil, centrada en el régimen de la propiedad inmobiliaria rústica, esta circunstancia se reflejaría en los primeros textos para el estudio universitario del Derecho Agrario y en los primaros programas de la asignatura.

Sobre la base de tales presupuestos resulta apreciable en el sistema de conocimientos el avance en los diferentes Programas hacia una noción cada vez más amplia sobre los temas que conforman el contenido a impartir, siendo así que se han ido incorporando nuevos temas que nos acercan a los nuevos rumbos del Derecho Agrario a nivel internacional y que derivan de la recepción de instrumentos internacionales, como ocurre con el tema ambiental en su relación con la actividad agropecuaria.

En otro sentido preciso es destacar la tendencia en la conformación del sistema de conocimientos del Derecho Agrario, que se contrapone a lo que tradicionalmente se había venido observando en otras asignaturas del Plan de la carrera de Derecho. Como ya se ha señalado, el Plan de Estudios C, constituyó la expresión de la voluntad académica encaminada a superar los enfoques R. Fac. Dir. UFG, V. 37, n. 1, p. 117 - 142, jan. / jun. 2013 
normativistas, formalistas y positivistas en los estudios jurídicos y sustituirlos por una enseñanza de alto contenido científico, lo que pudiera resumirse en la frase, del destacado profesor Julio Fernández Bulté, al conducir la elaboración del aludido Plan de Estudios: "Enseñar Derecho y no legislación”.

A este respecto se puede visualizar que el Derecho Agrario, incluyó en sus programa analítico desde el Plan B, en su sistema de conocimiento toda una serie de aspectos que iban más allá del examen de la legislación agraria, pertenecientes a la Filosofía, la Economía Política, la Economía de la Agricultura, la Sociología Rural, la Ecología, la Historia y otras ciencias,

De lo cual se colige que la impronta positivista no ha sido aquí tan marcada como lo ha sido en la mayoría de las asignaturas del currículo jurídico .en las últimas décadas en Cuba.

En cuanto a la forma de agrupar los contenidos, se pueden visualizar matices diferentes en los diferentes programas de las Universidades. En la UO, de acuerdo con criterios debatidos y consensuados en las diferentes actividades metodológicas de los últimos años, se defiende la idea de agrupar los contenidos en pocos temas, buscando mayores niveles de integración de los contenidos lo que favorece la enseñanza, lo cual puede apreciarse en las Tabla 3,4 y 5.

Tabla 3. Número de Temas

\begin{tabular}{|l|l|}
\hline \multicolumn{1}{|c|}{$\begin{array}{c}\text { Plan de } \\
\text { Estudios }\end{array}$} & \multicolumn{1}{c|}{$\begin{array}{c}\text { Cantidad de } \\
\text { Temas }\end{array}$} \\
\hline B UH & 10 \\
\hline B UO & 8 \\
\hline C UH & 8 \\
\hline C UO & 5 \\
\hline $\begin{array}{l}\text { C UH } \\
\text { Perfeccionado }\end{array}$ & 7 \\
\hline $\begin{array}{l}\text { C UO } \\
\text { Perfeccionado }\end{array}$ & 4 \\
\hline
\end{tabular}


Tabla 4 Temas y Contenidos. Plan B.

\begin{tabular}{|l|l|}
\hline Plan de Estudio & Temas \\
\hline B UH & 1. Teoría Marxista sobre la cuestión agraria. \\
2. Derecho Agrario. \\
3. Reforma Agraria. \\
4. La organización agropecuaria en Cuba. \\
5. Organismos relacionados con la agricultura \\
6. Organización de la producción agraria estatal. \\
7. Organización de los productores privados. \\
8. Crédito y Comercialización. \\
9. Nuevas formas de la producción agropecuaria \\
10. El Derecho Comparado \\
\hline 1. Teoría Marxista sobre la cuestión agraria. \\
2. Derecho Agrario. \\
3. Reforma Agraria. \\
5. Organización de la producción agraria estatal. \\
6. Organización de los productores privados. \\
7. Crédito y Comercialización. \\
8. Las Cooperativas agropecuarias. Acciones protectoras \\
del derecho de propiedad sobre la tierra y bienes \\
agropecuarios.
\end{tabular}

Tabla. Temas y Contenidos. Plan C y Plan C perfeccionados.

\begin{tabular}{|l|l|}
\hline Plan de Estudio & Temas \\
\hline C UH & 1. Teoría Marxista sobre la cuestión agraria. \\
& 2. Derecho Agrario. \\
& 3. Reforma Agraria. \\
& 4. Producción agropecuaria Estatal. \\
\hline
\end{tabular}




\begin{tabular}{|c|c|}
\hline & $\begin{array}{l}\text { 5. Pequeños Agricultores. } \\
\text { 6. Tenencia, uso y traspaso de la tierra. } \\
\text { 7. Cooperativas Agropecuarias. } \\
\text { 8. Registro de la Tierra y de semovientes }\end{array}$ \\
\hline C UO & $\begin{array}{l}\text { 1. Derecho Agrario. } \\
\text { 2. Fundamentos de la Política Agraria. } \\
\text { 3. El Derecho de propiedad de los agricultores pequeños } \\
\text { 4 El Derecho de propiedad de las cooperativas. } \\
\text { 5. El papel del Minagri en relación con los agricultores } \\
\text { pequeños y cooperativas.. }\end{array}$ \\
\hline C UH Perfeccionado & $\begin{array}{l}\text { 1. Derecho Agrario. } \\
\text { 2. Reforma Agraria en Cuba. } \\
\text { 3. Los sujetos de la relación jurídica agraria } \\
\text { 4. Objeto de la relación jurídica agraria. } \\
\text { 5. Infracciones y sistema contravencional en la actividad } \\
\text { agropecuaria. } \\
\text { 6. Comercialización, crédito, seguros y tributación. } \\
\text { 7. Protección al medio ambiente en la actividad } \\
\text { agropecuaria. }\end{array}$ \\
\hline C UO perfeccionado & $\begin{array}{l}\text { 1. Derecho Agrario fundamentos teóricos y antecedentes } \\
\text { históricos. } \\
\text { 2. El Derecho de propiedad de los agricultores pequeños } \\
3 \text { El Derecho de propiedad de las cooperativas. } \\
\text { 4. El papel del Minagri en relación con los agricultores } \\
\text { pequeños y cooperativas }\end{array}$ \\
\hline
\end{tabular}

El programa analítico actual de la asignatura, refleja el trabajo de los colectivos metodológicos a nivel de facultad y Departamento en la UO, la maduración de experiencias en la didáctica especial de esta asignatura, así como la introducción de los resultados de las investigaciones científicas en este campo, visualizándose la concurrencia de aspectos teóricos, normativos, de Derecho Comparado, sociológicos, político-ideológicos, filosóficos e históricos, tratando de reservar espacios R. Fac. Dir. UFG, V. 37, n. 1, p. 117 - 142, jan. / jun. 2013 
adecuados para cada uno de estos componentes y de lograr niveles satisfactorios de formación de valores en los estudiantes y de su formación integral.

\subsection{El Plan temático y la distribución del fondo de tiempo por actividades.}

En general han existido variaciones en el fondo horario previsto para la signatura y la dinámica de cambios en el fondo de tiempo total de la asignatura, se ha manifestado en una distribución diferente del fondo horario por temas y tipos de actividades en las facultades de Derecho.

La diferencia más apreciable reside en que en el Plan de la UO, se ha ido dedicando cada vez menor tiempo a las Conferencias, ampliándose el fondo a favor de los seminarios y clase prácticas, que alcanzan el último Plan de Estudio el 80\%, ello se justifica a partir de la recepción el la facultad de Derecho de la UO, de las nuevas corrientes pedagógicas, que asignan un protagonismo mayor al alumno en el proceso de enseñanza aprendizaje, mediante la búsqueda y construcción del conocimiento y el trabajo en colectivo. Lo cual se ilustra en la tabla a continuación.

Tabla 6 Distribución del fondo horario por tipos de actividades.

\begin{tabular}{|l|l|l|l|l|l|}
\hline \multicolumn{1}{|c|}{ Plan de } & \multicolumn{1}{|c|}{$\begin{array}{c}\text { Total del } \\
\text { fondo } \\
\text { Horario }\end{array}$} & \multicolumn{1}{|c|}{ Conferencias } & Seminarios & \multicolumn{1}{c|}{$\begin{array}{c}\text { Clases } \\
\text { Prácticas }\end{array}$} & $\begin{array}{c}\text { Clases } \\
\text { Mixtas }\end{array}$ \\
\hline B UH & 64 & 32 & 20 & 12 & \\
\hline B UO & 64 & 32 & 20 & 12 & \\
\hline C UH & 52 & 26 & 12 & 2 & 12 \\
\hline C UO & 42 & 10 & 18 & 8 & 6 \\
\hline $\begin{array}{l}\text { C UH } \\
\text { Perfeccionado }\end{array}$ & 50 & 24 & 8 & 6 & 6 \\
\hline $\begin{array}{l}\text { C UO } \\
\text { Perfeccionado }\end{array}$ & 50 & 10 & 20 & 14 & \\
\hline
\end{tabular}




\subsection{El sistema evaluativo}

Los cambios necesariamente constantes en el sistema de evaluación de las asignaturas ha sido un derivado de los análisis de la Comisión Nacional de la carrera de Derecho y del colectivo de carrera en la propia Facultad de Derecho de la UO, la decisión en el contexto del Plan C, fue la de suprimirle en Examen Final al Derecho Agrario. En consecuencia la concepción del sistema evaluativo dentro del Programa Analítico ha sido la de desplazar el interés de la Evaluación Final, hacia las Pruebas Parciales y las evaluaciones sistemáticas en clase, tal t como se muestra en la Tabla 6.

Tabla 6. Sistema Evaluativo

\begin{tabular}{|c|c|c|c|c|c|}
\hline $\begin{array}{c}\text { Plan de } \\
\text { Estudios }\end{array}$ & Exam & Final & $\begin{array}{c}\text { Pruebas } \\
\text { parciales }\end{array}$ & $\begin{array}{c}\text { Trabajos en } \\
\text { Clases }\end{array}$ & $\begin{array}{c}\text { Trabajos } \\
\text { Extractases }\end{array}$ \\
\hline B UH & $\begin{array}{l}\text { Escrito } \\
\mathrm{X}\end{array}$ & Oral & 1 & 2 & 1 \\
\hline B UO & $X$ & & 1 & 1 & 1 \\
\hline $\mathrm{C} \mathrm{UH}$ & $X$ & & 1 & & \\
\hline $\mathrm{C} \mathrm{UO}$ & $X$ & & 2 & & 1 \\
\hline $\begin{array}{l}\text { C UH } \\
\text { Perfeccionado }\end{array}$ & $X$ & & 2 & & \\
\hline $\begin{array}{l}\text { C UO } \\
\text { Perfeccionado }\end{array}$ & No & & 1 & & 1 \\
\hline
\end{tabular}

Se ha arribado así a una concepción en la que se atiende en mayor medida al principio de objetividad en la evaluación, siendo así que la Prueba Parcial centra su atención en el dominio de aspectos conceptuales y teóricos en general y en la capacidad para aplicar conceptos, se toma en consideración que se trata de un alumno que está cursando el cuarto año, que debe alcanzar niveles concretos en el desarrollo de habilidades profesionales,

\subsection{La Bibliografía}


Por lo general los Programas Analíticos de las Asignaturas han incluido la referencia a la literatura imprescindible para el estudio por parte del alumno.

Al examinar los Programas Analíticos que ha tenido la asignatura de Derecho Agrario son apreciables algunas dificultades en este sentido. En el caso del programa de la asignatura, para el Plan $\mathrm{B}$, estas indicaciones se limitaban a señalar la bibliografía básica integrada por tres textos, lo que claro está reflejaba la situación bibliográfica existente en el país en ese momento, pero ello era expresivo de la concepción del estudio de la asignatura a partir de un texto básico, normalmente un manual.

En el Manual, en el que se desarrollaban de manera secuente los contenidos del programa, se le asignaba lógicamente un amplio espacio al tratamiento del tema acerca de la Reforma Agraria en Cuba y al proceso de cooperativización y de desarrollo del marco normativo sobre las cooperativas, ya hemos comentado que para ese período a penas se había producido literatura cubana acerca de la teoría del Derecho Agrario y los estudios comparados sobre los institutos agrarios creación de. Obvio resulta decir entonces que el actual programa de la asignatura revela los avances alcanzados por el Derecho Agrario cubano, en el aspecto doctrinal y normativo, es apreciable la producción de literatura nacional al alcance de los estudiantes, que si bien aún no sería suficiente coloca a los estudiantes de Derecho en una situación mucho más favorable para el aprendizaje, al mismo tiempo se ha ido produciendo la recepción en los programas de obras procedentes de autores extranjeros.

\section{La Enseñanza de la Asignatura de Derecho Agrario a Través del Modelo de Enseñanza no Presencial y Semipresencial}

\subsection{El estudio de la asignatura de Derecho Agrario en la modalidad a distancia}

A finales de los años 70, habían comenzado lo estudios universitarios de Derecho a través del Modelo de enseñanza a Distancia, no presencial. Sin embargo, tal y como ocurrió con la enseñanza presencial, en el primer Plan de Estudios, o se en el Plan A, no figuraba el Derecho Agrario en el currículo, es con el Plan B, que se introduce la asignatura de Derecho Agrario. 
A este respecto resulta necesario aclarar, que a diferencia de lo previsto para el modelo presencial, aquí en el modelo no presencial, ha regido el principio de la centralización, concretado en que el Programa de la Asignatura, viene elaborado por la Universidad de La Habana como centro rector en la carrera de Derecho, se debe tomar en consideración que cada programa ha regido durante períodos largos de 5 a 10 años, esta situación lógicamente conduce a que frecuentemente se haya estando impartiendo un Programa con contenidos desactualizados, dada la dinámica de los cambios normativos, muy frecuentes y profundos en este campo, esta afectación se producía simultáneamente en el manual de la asignatura. Esto generaría múltiples problemas para los estudiantes a la hora de estudiar los contenidos y realizar los exámenes, pues para los profesores de Derecho, no es admisible una respuesta basada en una legislación ya derogada. ${ }^{15}$ En ocasiones esto ha podido ser resuelto mediante algunos materiales didácticos, y las consultas que el profesor formal o informalmente brinda a los alumnos, previo al examen o cuando el alumno impugnaba la nota recibida y el profesor le explicaba lo ocurrido. Pero esta ha sido sin dudas una debilidad de esos programas analíticos de Derecho Agrario.

Una debilidad de los programas analíticos de las asignaturas en la modalidad de estudios a distancia tanto en los Planes B, como en el C, ha sido la ausencia de actividades encaminadas a la formación de las habilidades profesionales de interpretar y aplicar bien la legislación, enfatizándose el estudio memorístico de los conceptos y los contenidos de la legislación.

La ubicación de la asignatura de Derecho Agrario, como parte del Ciclo III, último ciclo de asignaturas del currículo, ha sido correcta dados los conocimientos precedentes que requiere para su estudio. $^{16}$

En cuanto al sistema de conocimientos en los diferentes Programas de Derecho Agrario, para la enseñanza a distancia, no se aprecian grandes diferencia entre ellos, no obstante cabe hacer notar la presencia de un tema en el Plan B, acerca de los Fundamentos ideológicos y teóricos del Derecho Agrario cubano (lo que remitía al análisis del pensamiento filosófico marxista leninista y del pensamiento político jurídico cubano acerca de la cuestión agraria) lo cual parecía una idea acertada, sin embargo el tema fue suprimido del Plan C, lo que puede comprobarse en la Tabla 7.

\section{Tabla 7. Temas y Contenidos en los Planes B y C. Estudios a Distancia.}

R. Fac. Dir. UFG, V. 37, n. 1, p. 117 - 142, jan. / jun. 2013

ISSN 0101-7187 


\begin{tabular}{|l|l|}
\hline Plan de Estudio & Temas \\
\hline B & 1. Teoría Marxista sobre la cuestión agraria. \\
2. Derecho Agrario. \\
3. Reforma Agraria. \\
4. La organización del sector agrícola estatal. \\
\\
5. Los agricultores pequeños, tenencia, uso y traspaso de \\
la tierra. \\
6. Las cooperativas agropecuarias. \\
7. La actividad registral en materia agraria> el registro de \\
semovientes y el de tenencia de la tierra. \\
\hline C \\
$\begin{array}{l}\text { 1. Derecho Agrario. } \\
\text { 2. Reforma Agraria. } \\
\text { 3. La producción agrícola estatal. } \\
\text { 4. Los pequeños agricultores. La tenencia, uso y traspaso } \\
\text { de la tierra de los agricultores pequeños. } \\
\text { 5. Las cooperativas agropecuarias. } \\
\text { 6. La actividad registral en materia agraria: el registro de } \\
\text { semovientes y el de la tierra. } \\
\end{array}$ \\
\hline
\end{tabular}

En cuanto a los objetivos: en el Plan B, se trataba de objetivos demasiado abstractos y generales, en cambio en Plan C, aunque siguen siendo objetivos generales, se proyectan de manera que ofrecen una mayor claridad acerca de los propósitos por cada tema del Programa.

En ambos Programas, de Plan B y Plan C, se carece de las indicaciones metodológicas para el estudio de la asignatura, acerca de qué y como estudiar.

En relación con la bibliografía, sólo se indica en los Programas el estudio del manual y de algunas legislaciones, que como ya se hay dicho había devenido desactualizada e insuficiente, hasta fecha reciente 
Como ya se ha señalado se carece en el programa de proyección en cuanto a procurar el desarrollo de habilidades relacionadas con la interpretación y aplicación de la legislación agraria. ${ }^{17}$

2.2 La enseñanza del Derecho Agrario a través del Modelo de enseñaza semipresencial, denominado como de la Universalización o de la SUM (sedes universitarias municipales) ${ }^{18}$

La enseñanza del Derecho, a través del modelo de la universalización, comenzó en el año 2001 en la Universidad de La Habana, y en el 2002 en la UO y todo el resto del país. Las notas distintivas de este modelo residen en que la enseñanza aquí se desarrolla de manera semipresencial y una mayor descentralización que en la enseñanza a distancia sobre todo en lo que se refiere a la evaluación. En cuanto a la forma de docencia, ello supone el predominio de la clase encuentro donde el profesor, orienta el estudio de los contenidos, ofrece indicaciones metodológicas, orienta el estudio y la realización de trabajos de investigación o de prácticas profesionales para la gestión o construcción del conocimiento por parte del alumno, chequea y evalúa el aprendizaje.

Las fortalezas de esta modalidad consisten fundamentalmente en las mayores posibilidades de desarrollo de habilidades para el autoaprendizaje y en el hecho de que los profesores y tutores son profesionales del Derecho que poseen elevadas competencias en la práctica profesional, lo que puede favorecer tanto el aprendizaje como la formación de habilidades y valores.

En cuanto a la ubicación, asignatura de Derecho Agrario, se ha situado en el cuarto año de la carrera, lo que nos parece adecuado por las razones ya apuntadas, pero obviamente no podremos ser muy pródigos en esta parte de la exposición pues, ello significa que la asignatura, apenas la hemos impartido dos veces a través de este modelo pedagógico y las experiencias resultan limitadas.

El fondo de tiempo para las actividades frente al profesor es de 16 horas, previsto para el desarrollo de las aludidas, se supone que para las clases encuentro.

Los objetivos generales, han sido redactados de manera que se integran los instructivos y los educativos, criterio que nos parece correcto. 
En cuanto a las indicaciones metodológicas se aprecia una cuestión con la cual se pudiera discrepar, si la modalidad de enseñanza es semipresencial y la forma de docencia deben ser las clases encuentro, no resulta lógico que se hable aquí de que se impartirán conferencia, clases prácticas y seminarios, cual si fuera en el modelo de enseñanza presencial.

En cuanto a las habilidades, la que se describe como la número tres y que enuncia, "Desarrollar hábitos y habilidades acordes con las concepciones teóricas y prácticas en que se fundamenta la legislación agraria", ofrece bastante vaguedad con respecto a qué es lo que se quiere lograr.

El sistema de conocimientos previstos en general resulta adecuado, sin embargo se aprecian algunas cuestiones que deben ser revisadas:

- El sistema de conocimientos sólo se refiere a las infracciones y contravenciones del régimen de uso y explotación de la tierra, quedando sin examen todo el amplio número de infracciones que tienen que ver con la actividad agropecuaria en materia como: forestal, ganadería, tractores, flora y fauna silvestre, sanidad vegetal, medicina veterinaria, semillas, suelos, riego y otras.

- La inclusión de un epígrafe sobre la enseñanza del Derecho Agrario, aunque sin dudas resulta interesante, no parece guardar relación con el sistema de conocimientos y habilidades previstos en el programa.

- La inclusión del epígrafe sobre el estudio de las Unidades Básicas de Producción Cooperativa, dentro del Tema acerca de la Organización Estatal de la Agricultura, puede ser cuestionable, posee pocos argumentos a favor, habida cuenta de que la definición legal no la califica dentro de las formas estatales de producción agrícola.

Los objetivos específicos por temas pudieran perfeccionarse en su redacción, sobre todo en lo que se refiere a su verbo rector, ya que se abusa del verbo conocer, que limita el aprendizaje a sus niveles y etapas más incipientes, en otros casos se emplea el verbo analizar, lo que precisa muy poco en cuanto a la transformación a lograr en el alumno, con el estudio del tema.

En cuanto al sistema de evaluación se aprecia como deficiencia, la insuficiente presencia del componente investigativo, ya que solo se prevé la realización por los alumnos de un trabajo R. Fac. Dir. UFG, V. 37, n. 1, p. 117 - 142, jan. / jun. 2013 ISSN 0101-7187 
investigativo individual, sobre el tema II, sin expresarse su objetivo, su metodología (si se trata de resumir fuentes legales o es otro el propósito).

Por último cabe advertir, que el hecho de que el programa sea elaborado por la Universidad de La Habana de manera centralizada para todo el país, le imprime cierta rigidez, contradictoria con la flexibilidad propia de esta modalidad de estudios, lo que dificultaría la respuesta oportuna ante la necesidad de suprimir o incluir nuevos contenidos, como respuesta a los cambios institucionales o legales que pudieran introducirse.

\section{Conclusiones}

Primera: La impartición del Derecho Agrario como asignatura autónoma, de carácter obligatorio en los planes de estudio de la carrera de Derecho en Cuba, constituye un logro indiscutible en cuanto a la formación de los juristas.

Debe conservarse y desarrollarse principalmente en aquellas provincias en las que la producción agropecuaria tiene un peso fundamental.

Segunda: Los actuales programas analíticos de la signatura, para las diferentes modalidades de estudio revelan los avances alcanzados por el Derecho Agrario cubano, en el aspecto doctrinal y normativo, es apreciable en este sentido la remisión al estudio de una literatura nacional al alcance de los estudiantes, que si bien aún no sería suficiente coloca a los estudiantes de Derecho en una situación mucho más favorable para el aprendizaje, al mismo tiempo se ha ido produciendo la recepción en los programas de obras procedentes de autores extranjeros.

Tercera: Los actuales programas analíticos de la asignatura de Derecho Agrario para su impartición en la enseñanza presencial, en general resultan adecuados al nivel de las experiencias pedagógicas acumuladas en este campo, a las necesidades sociales actuales, apreciándose algunas deficiencias en cuanto al sistema de habilidades y las indicaciones metodológicas para su estudio. 
Cuarta: Existen algunas deficiencias en el programa analítico de la asignatura, en la modalidad de estudios a distancia consistentes en:

a) La ausencia de un tema acerca de los fundamentos ideológicos y teóricos del Derecho Agrario cubano, lo cual no posibilita al alumno comprender correctamente las fuentes de la política agraria del Estado cubano.

b) Carencia de proyección en cuanto a procurar el desarrollo de habilidades relacionadas con la interpretación y aplicación de la legislación agraria

c) Se omiten las indicaciones metodológicas para el estudio de la asignatura, acerca de qué y como estudiar.

Quinta: Existen algunas deficiencias en el programa analítico de la asignatura en la modalidad semipresencial, consistentes:

a) En cuanto a las habilidades, se aprecia cierta vaguedad con respecto a qué es lo que se quiere lograr.

b) El sistema de conocimientos sólo se refiere a las infracciones y contravenciones del régimen de uso y explotación de la tierra y la inclusión del epígrafe sobre el estudio de las Unidades Básicas de Producción Cooperativa, dentro del tema acerca de la Organización Estatal de la Agricultura, puede ser cuestionable, habida cuenta de que la definición legal no la califica dentro de las formas estatales de producción agrícola.

c) Los objetivos específicos por temas abusan del verbo conocer, que limita el aprendizaje a sus niveles y etapas más incipientes, en otros casos se emplea el verbo analizar, lo que precisa muy poco en cuanto a la transformación a lograr en el alumno, con el estudio del tema.

d) En cuanto al sistema de evaluación existe insuficiente presencia del componente investigativo, ya que sólo se prevé la realización por los alumnos de un trabajo investigativo individual, sobre el tema II que está referido al marco jurídico de las distintas formas organizativas de la producción agrícola.

\section{Recomendaciones}

Primera: (A la Sociedad Cubana de Derecho Agrario y a las Facultades de Derecho) La impartición del Derecho Agrario como asignatura autónoma, de carácter obligatorio en los planes de R. Fac. Dir. UFG, V. 37, n. 1, p. 117 - 142, jan. / jun. 2013 
estudio de la carrera de Derecho en Cuba, en todas las modalidades de estudio, debe conservarse y desarrollarse principalmente en aquellas provincias en las que la producción agropecuaria tiene un peso fundamental.

Segunda: (A las Facultades de Derecho) Los actuales Programas Analíticos, aunque en general resultan adecuados, poseen algunos aspectos aludidos en las conclusiones que requieren ser revisados.

\section{Referencias}

BALLARÍN MARCIAL, Alberto. Derecho Agrario, En: Revista de Derecho Privado. Madrid: 1978.

DORTA DUQUE, Manuel. Proyecto de Código Cubano de Reforma Agraria , La Habana, 1956.

GELSI BIDART, Adolfo. Perfiles Sistemáticos y Líneas de Tendencias del Derecho Agrario. Revista Derecho y Reforma Agraria.16/1989. Instituto Iberoamericano de Derecho Agrario y Reforma Agraria. (IIDARA). Mérida. Venezuela.

MC CORMACK BÉCQUER, Maritza y otros. Temas de Derecho Agrario, Ed. Félix Varela, La Habana, 2007

PAVO ACOSTA, Rolando. Acerca de la Autonomía del Derecho Agrario, Revista Barco de Papel, Edición Especial, Memorias de la Conferencia Científica Internacional sobre el Derecho, Mayagüez, Puerto Rico, 1998.

RUIZ MASSIEU, Mario. Temas de Derecho Agrario Revolucionario, Instituto de Investigaciones Jurídica, UNAM, México, 1987, pp. y ver PAVO ACOSTA, Rolando, El Derecho Agrario la Integración de América Latina, Revista Correo de la ADIA, Numero 9, Año 4, Octubre de 1997, Santo Domingo, República Dominicana.

1 No resulta despreciable en el examen de esta cuestión, la influencia del hecho de que la mayoría de los juristas que en los últimos 10 años han obtenido el grado académico de doctor en ciencias jurídicas y que por tanto ocupan hoy cargos de dirección docente en las Universidades, han recibido superación profesional y académica en Europa, fundamentalmente en España.

${ }^{2}$ Ver PAVO ACOSTA, Rolando: Acerca de la Autonomía del Derecho Agrario, Revista Barco de Papel, Edición Especial, Memorias de la Conferencia Científica Internacional sobre el Derecho, Mayagüez, Puerto Rico, 1998.

${ }^{3}$ No es ésta la oportunidad para enarbolar posiciones que exacerben las diferencias y contradicciones, pero resulta evidente que todavía la construcción doctrinal, científica y normativa del país, en toda una serie de espacios, se realiza con un predominio de enfoques que no toman consideración las diferencias entre la capital y el resto del país, esta es una cuestión en la cual se ha ido avanzando, pero se debe seguir insistiendo ante lo necesario de lograr una unidad que al mismo tiempo considere la diversidad. 
${ }^{4}$ Ver RUIZ MASSIEU, Mario: Temas de Derecho Agrario Revolucionario, Instituto de Investigaciones Jurídica, UNAM, México, 1987, pp. y ver PAVO ACOSTA, Rolando, El Derecho Agrario la Integración de América Latina, Revista Correo de la ADIA, Numero 9, Año 4, Octubre de 1997, Santo Domingo, República Dominicana, pp. 9-10 y 34.

5 Así, por ejemplo, en la Universidad Castilla La Mancha se imparte en la carrera de Derecho la asignatura Derecho Agrario y Política Agraria, por la docente, Dra. Ana Carretero García, en la Universidad de Sevilla se imparte el Derecho Agrario por el Profesor Dr. Juan Ignacio Reales Espina.

${ }^{6}$ Véase GELSI BIDART, Adolfo. Perfiles Sistemáticos y Líneas de Tendencias del Derecho Agrario. Revista Derecho y Reforma Agraria.16/1989. Instituto Iberoamericano de Derecho Agrario y Reforma Agraria. (IIDARA). Mérida. Venezuela, pp. 51-86. Este criterio ha sido corroborado recientemente por los autores con la información brindada por las agraristas argentinas Nancy Malanos, María Adriana Victoria y Alba Esther de Bianchetti.

${ }^{7}$ Véase VIVANCO, Antonio. El Derecho Agrario en Argentina y sus corrientes doctrinarias. En: Revista del IIDARA No.20/91, pp.17-26

${ }^{8}$ Véase RUIZ MASSIEU, Mario. Derecho Agrario Revolucionario. UNAM. México. 1987, p.47- 48.

${ }^{9}$ BALLARÍN MARCIAL, Alberto, Derecho Agrario, En: Revista de Derecho Privado. Madrid.1978, p.414.

${ }^{10}$ VI-Conferencia de la UDUAL. Universidad de Externado, Bogotá. Colombia. 1976. UDUAL.

11 En el curso 82-82, se inicia la impartición del Plan de Estudio B, en el curso 90-91 inicia el Plan de Estudio C y en el curso 2000-2001 inicia el denominado Plan C Perfeccionado. XIII, L.

12 Ver DORTA DUQUE, Manuel: Proyecto de Código Cubano de Reforma Agraria, La Habana, 1956, Prefacio p.

${ }^{13}$ Ver Mc Cormack Bécquer, Maritza y otros: Temas de Derecho Agrario, Ed. Félix Varela, La Habana, 2007, pp. 19 y 20 .

14 La inclusión del Derecho Agrario como parte del Plan de Estudio B para la carrera de Derecho y la posterior elaboración e implantación del Programa Analítico de la asignatura y su impartición, se debió a la importante contribución del entonces profesor de la Universidad de la Universidad de La Habana Lic. Cratilio Navarrete Acevedo y del Dr. Juan Mir Pérez, profesor en la Universidad de Oriente. Luego la labor de elaboración de los Programas correspondientes a la asignatura en las Universidades de La Habana y Santiago de Cuba, para los Planes de Estudio C y C Perfeccionado, fue asumida por los profesores Dra. Maritza MC Cormack y Dr. Rolando Pavó, respectivamente.

15 Esta situación, como ya se ha expresado, no resultaba insoluble en la enseñanza presencial pues la relativa descentralización, permite al profesor en cada Universidad modificar el programa y actualizarlo y poner a disposición de los alumnos la nueva literatura actualizada elaborada por él.

16 Ver Programas. Ciclo III. Carrera de Derecho. Facultad de Enseñanza a Distancia. Universidad de La Habana. 2003, p. 2-6.

${ }^{17}$ Con respecto a todo lo señalado anteriormente, se estima pertinente no derivar luego recomendaciones encaminadas a perfeccionar este Programa Analítico para el modelo de enseñanza a distancia, comoquiera que se trata de un modelo en fase de liquidación, que se ha planteado extinguir, al ser absorbido completamente a corto plazo por la enseñanza semipresencial.

${ }^{18}$ En cuanto al modelo semipresencial, cabe hacer la salvedad que entre mediados de los 70 y hasta mediados de los 80, existió esta modelo, a través de la modalidad que se denominó entonces como cursos por encuentro, para trabajadores, pero el plan de estudios para esa modalidad, no incluyó a la asignatura de Derecho Agrario, graduándose los últimos estudiantes mediante esta modalidad en la Universidad de Oriente en 1987, y por lo tanto no nos detenemos en el análisis de esta modalidad de estudios de Derecho. 